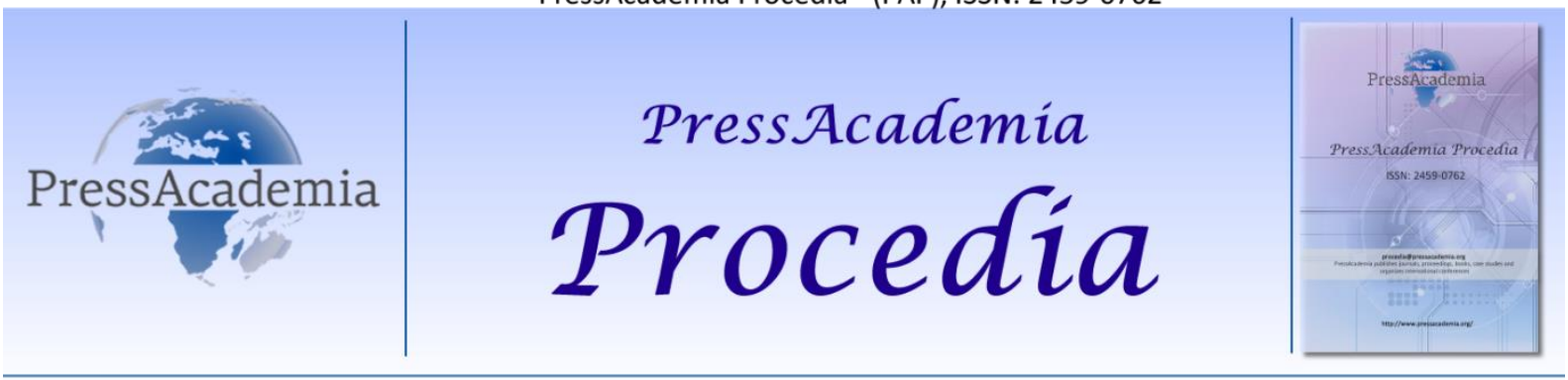

2nd World Conference on Technology, Innovation and Entrepreneurship

May 12-14, 2017, Istanbul, Turkey. Edited by Sefer Şener

\title{
DETERMINATION OF ENHANCEMENT EFFECTS AT THE COMPTON TO COHERENT SCATTERING INTENSITIES X-RAYS USING FE-CU AND FE-ZN BINARY COMPOUNDS WITH WDXRF
}

\author{
DOI: 10.17261/Pressacademia.2017.620 \\ PAP-WCTIE-V.5-2017(58)-p.431-433
}

\author{
Elif Boydas ${ }^{1}$ Demet $_{\text {Yilmaz }}{ }^{2}$ Mustafa Gokalp Boydas ${ }^{3}$ \\ ${ }^{1}$ Atatürk University .ebaydas@atauni.edu.tr \\ ${ }^{2}$ Atatürk University. ddemir@atauni.edu.tr \\ ${ }^{3}$ Atatürk University .mboydas@atauni.edu.tr
}

\begin{abstract}
Matrix enhancement effects have been examined with scattered X-rays by using WDXRF system for Fe-Cu and Fe-Zn binary compounds. Fe as analyte and elements which some of them the $\mathrm{K}$ absorption edge just below the characteristic $\mathrm{K} \mathrm{X}$-ray energies of the analit (Fe) as matrix have been chosen. Pure and binary with Fe ratio ranging from $10 \%$ to $90 \%$ samples were prepared with these elements. These samples have been analyzed in WDXRF system. At the end of measurement, the coherent and Compton scattering X-rays data of Rh K $\alpha$ Xrays from these samples have been obtained. Matrix enhancement effects have been examined by calculating coherent / Compton scattered X-rays intensity ratio.
\end{abstract}

Keywords: Analyte, enhancement, compton scattering, coherent scattering , x-rays

\section{INTRODUCTION}

Compton scattering is one of the methods giving information about the electronic structure, electronic momentum distribution and the wave functions of atoms, molecules and solids. Compton scattering is the scattering of a photon of high energy from an electron considered

being free and stationary or from an atomic electron whose binding energy is small compared with the incident photon energy. X-ray scattering techniques are nondestructive and often have a great advantage over the traditional chemical techniques used for determination of the effective atomic number of samples of environmental, biological, agricultural, industrial and medical interest. The $3 \mathrm{~d}$ transition metals and their alloys have played an important role in the development of modern technology, and knowledge of their physical parameters such as mass attenuation coefficients, total atomic and electronic cross-sections, and effective atomic and electron numbers is very important for understanding their physical properties.

X-ray scattering techniques are nondestructive and often have a great advantage over the traditional chemical techniques used for determination.of the effective atomic number of samples of environmental, biological, agricultural, industrial and medical interest. Han et al (2009) shows that the $3 d$ transition metals and their alloys have played an important role in the development of modern technology, and knowledge of their physical parameters such as mass attenuation coefficients, total atomic and electronic cross-sections, and effective atomic and electron numbers is very important for understanding their physical properties. Speller et al. (1991) have introduced applications of the coherent and Compton scattering in the fields such as medicine and biology. Webster et al (1985) have measured the coherent to Compton scattering intensity ratio in order to assess trabecular bone mineral content. Cooper et al (1982) examined sample composition change in alloys by using scattered intensity ratio. Gigante et al. (1985)have analyzed the metal alloys by Rayleigh to Compton ratios and X-ray fluorescence peaks in the 50-122 keV energy range. Shakeshaft et al (1997) have introduced applications of this technique in the fields of medicine and biology. 
In this study, We measured coharent and Compton peaks of binary systems. We examined enhancement effects of matrix on analyt by using coharent/Compton scattering area ratios.

\section{EXPERIMENTAL}

Fe was used as analyt. As a matrix were used elements such as $\mathrm{Cu}, \mathrm{Zn}$. While concentration of analyt gradually increased in steps of $10 \%$ from $10 \%$ to $\% 90$, that of matrix gradually decreased in steps of $10 \%$ from $90 \%$ to $\% 10$.Analyt and one of matrice were mixed these proportions. For example, this proportion can be such as $\mathrm{Fe}$ of $30 \%$ and $\mathrm{Ca}$ of $70 \%$ in binary systems. Compton and coharent peaks of this samples were measured by using wavelength-dispersive X-ray fluorescence (WDXRF) spectrometry.

\section{FINDINGS AND DISCUSSIONS}

$R(R=I c o h / I C)$ versus concentration of Fe in binary systems of Fe-Cu, Fe- $\mathrm{Zn}$ are given in Fig. 1-2. Thus this enhancement effects is observed in the $\mathrm{Zn}$ element with respect to the Fig.2. These dispersed X-rays of matrix elements might also excite the examining element (analyte) as well, be called the Matrix enhancement effects effect. Concluded that ratio of Icoh/Ic ascended in the position of decrement for all systems concentration of $\mathrm{Fe}$, while increment of other Matrix concentration. Increment in this ratio of these elements evident that could be an intensify impulse on Fe $\mathrm{K} X$-ray. In as much as $\mathrm{K}$ shell absorption edge of $\mathrm{Co}, \mathrm{Ni}, \mathrm{Cu}$ and $\mathrm{Zn}$ reside inside of short wavelength of Fe $\mathrm{K}$ edge. Accordingly these elements' primary $\mathrm{X}$ ray absorption is very narrow so that characteristic X-rays of these elements will be intensifying the Fe $\mathrm{K} X$-rays and can be considered as intensifying elements of previous stated elements.

Figure 1: $R(R=I c o h / I C)$ versus Concentration of Fe in Binary Systems of Fe-Cu

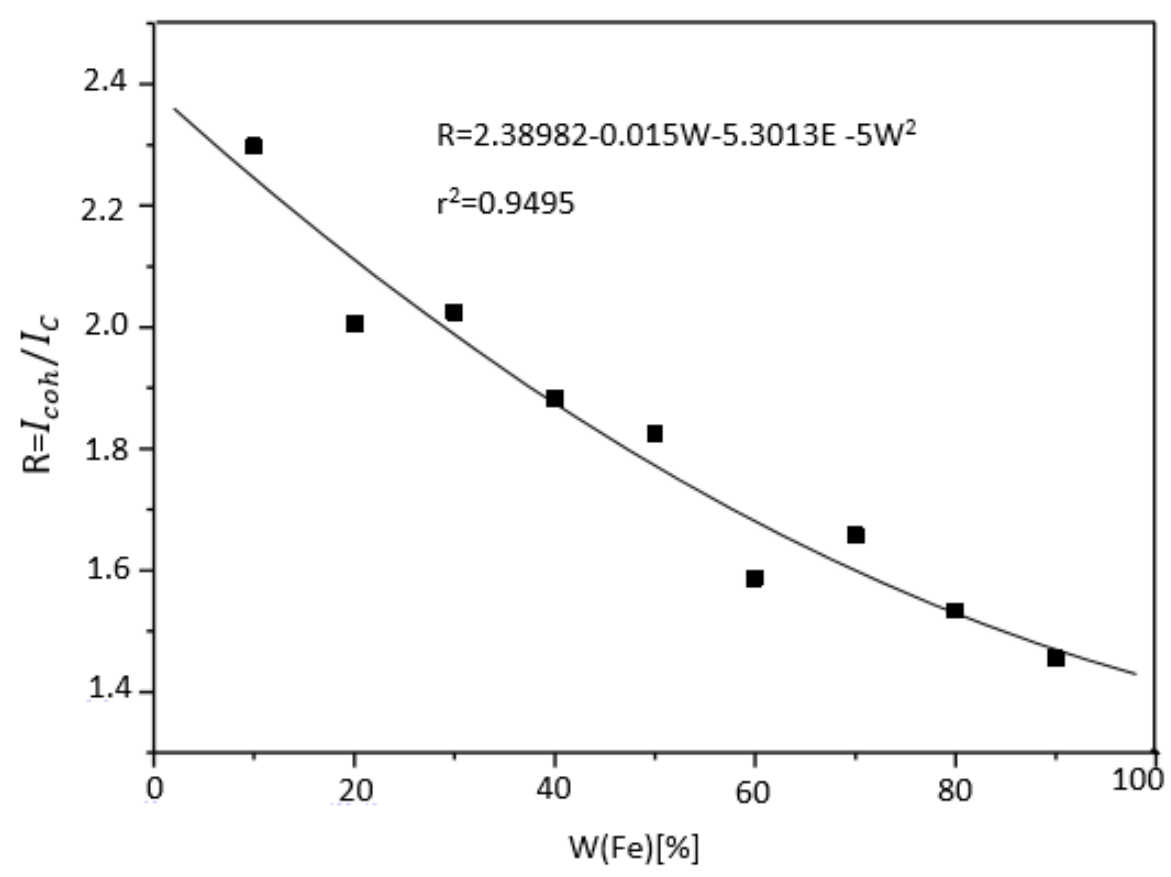


Figure 2: $R(R=I c o h / I C)$ versus Concentration of Fe in Binary Systems of Fe-Zn

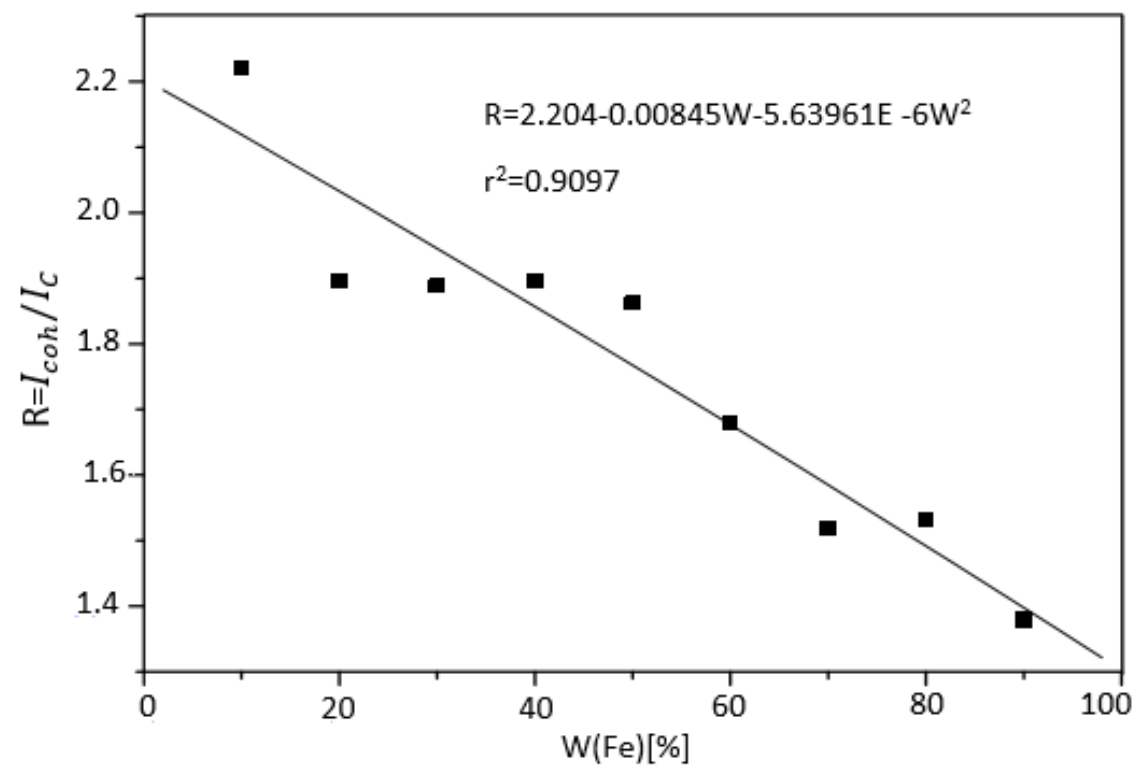

\section{CONCLUSION}

The aim of the present study is to determine enhancement effects using coherent / Compton scattered X-rays intensity ratio. Matrix enhancement effects have been examined with scattered X-rays by using WDXRF system for Fe-Cu and Fe-Zn binary compounds. the coherent and Compton scattering X-rays data of Rh $\mathrm{K} \alpha \mathrm{X}$-rays from these samples have been obtained. Matrix enhancement effects have been examined by calculating coherent / Compton scattered X-rays intensity ratio.

\section{REFERENCES}

Han, I., Demir, L. 2009. "Mass attenuation coefficients, effective atomic and electron numbers of Ti and Ni alloys",Radiation. Measurement, vol.44 no. 1 ,pp. $289-294$.

Speller, R. D., Horrocks, J. A. 1991," Photon scattering--a "new' source of information in medicine and biology?" Physics in Medicine and Biology .Vol.36, no.,pp.1.

Webster, D. J., Lillicrap, S. C.1985,"Coherent-Compton scattering for the assessment of bone mineral content using heavily filtered X-ray beams" ,Physics in Medicine and Biology, volume 30, no. 6, pp. 531-539.

Cooper,M.J., Rollason, A.J., Tuxworth, R.W. 1982," Gamma-ray scattering studies of composition variations in alloys" J. Phys. E: Sci. Instrum,vol.15, pp. 568-572

Gigante,G.E., . Pedraza, L.J, Sciuti, S. 1985," Analysis of metal alloys by Rayleigh to Compton ratios and x-ray fluorescence peaks in the 50 to 122 keV energy range" Nucl. Inst. Meth, vol.12, pp. 229-234

Shakeshaft,J., Morgan H.M., Lillicrap, S.C.1997, “Gamma-ray scattering for fat fraction measurement”, Physics in Medicine and Biology, vol. 42, no.7,pp. 1403-1413. 